\title{
Energy efficient scheme based on simultaneous transmission of the local decisions in cooperative spectrum sensing
}

\author{
Guilherme Pedro Aquino ${ }^{1}$, Dayan Adionel Guimarães ${ }^{1}$ \\ and Marco E. G. V. Cattaneo ${ }^{2}$ \\ ${ }^{1}$ National Institute of Telecommunications (Inatel) \\ Santa Rita do Sapucaí, MG - BRA \\ [e-mail: guilhermeaquino/dayan@inatel.br] \\ ${ }^{2}$ University of Hull \\ Hull, Yorkshire - UK \\ [e-mail: m.cattaneo@hull.ac.uk] \\ *Corresponding author: Guilherme Pedro Aquino
}

Received September 21, 2015; revised November 24, 2015; revised December 11, 2015;

accepted December 27, 2015; published March 31, 2016

\begin{abstract}
A common concern regarding cooperative spectrum sensing (CSS) schemes is the occupied bandwidth and the energy consumption during the transmissions of sensing information to the fusion center over the reporting control channels. This concern is intensified if the number of cooperating secondary users in the network is large. This article presents a new fusion strategy for a CSS scheme, aiming at increasing the energy efficiency of a recently proposed bandwidth-efficient fusion scheme. Analytical results and computational simulations unveil a high increase in energy efficiency when compared with the original approach, yet achieving better performances in some situations, and lower implementation complexity.
\end{abstract}

Keywords: Cognitive radio, cooperative spectrum sensing, fusion of sensor information, energy efficiency.

This work was partially supported by Finep, with resources from Funttel, Grant No. 01.14.0231.00, under the Radiocommunication Reference Center (Centro de Referência em Radiocomunicações - CRR) project of the National Institute of Telecommunications (Instituto Nacional de Telecomunicações - Inatel), Brazil. 


\section{Introduction}

The cognitive radio (CR) [1] concept has emerged as a candidate for mitigating the problem of spectrum scarcity faced by the wireless communication systems under the present policy of fixed allocation of the radiofrequency spectrum. This is done by allowing that unlicensed devices access a specific licensed band in periods of time or geographic areas in which it is unoccupied by the primary users (PU), which owns the right of using this band. These unlicensed devices are called cognitive users or secondary users (SU), and such an access method is usually referred to as an opportunistic spectrum access.

Before using a licensed band opportunistically, a CR must be capable of examining a portion of the spectrum, looking for the so-called white spaces (portions of unused spectrum). This spectrum sensing [2] capability is one of the main tasks of a CR. It will permit a CR to transmit its data without harm to the PU operation.

Due to the receiver noise, multipath fading and shadowing, the performance of the spectrum sensing when made by a single SU may be seriously degraded, hindering an accurate SU decision about the channel occupancy. The cooperative spectrum sensing (CSS) is capable of mitigating this problem [3]. In centralized CSS, a set of SUs sense the spectrum and send their sensing measurements or local decisions about channel occupancy to a fusion center (FC), where the global decision about the channel occupancy is made by combining the SU's measurements or decisions [4]. The transmission of the sensing information is normally made via dedicated report channels.

Bandwidth and energy efficiency are aspects of paramount importance in present wireless communication systems, but even more for the development of the future fifth-generation (5G), in which the cognitive radio concept plays an important role [5]. Within this context, a common concern regarding CSS schemes is the bandwidth and energy consumption during the transmissions of sensing information to the FC. This concern is intensified if the number of cooperating SUs is large, since more bandwidth and energy resources will be needed.

A number of attempts have been made for saving energy and bandwidth resources during the report of secondary user's decisions; see for example [6-12]. Besides the bandwidth efficiency, the energy efficiency is also an important aspect of the spectrum sensing, being considered for example in [13-22].

Recently, a novel bandwidth-efficient fusion scheme for CSS was proposed in [23], along with extended results and discussions in [24]. In this scheme, the cooperating secondary users transmit their local decision at the same time and in the same bandwidth to the fusion center. Consequently, it provides a significant reduction in the required bandwidth of the report channel when compared with systems that use some orthogonal transmission approach, like time or frequency division multiple access. Shortly, in the idea proposed in [23] each SU transmits its local decision on the channel occupancy to the FC using a binary phase shift keying (BPSK) signaling. Since all SUs send their BPSK symbols simultaneously and occupying the same frequency band, the received symbols at the FC add non-coherently. Under the $K$-out-of- $M$ rule, two sets of symbols are formed at the FC due to this non-coherency, one set being associated to $K$ or more SUs deciding in favor of the presence of the PU signal, and the other set being associated to less than $K$ SUs deciding for the presence of the PU signal. In [23] it is also proposed a computationally costly decision rule for discriminating these two sets. However, in [23] no attention has been directed to the energy consumption related to the decision reporting task. In other words, the new scheme of [23] 
increases the spectrum efficiency, but does not provide any means for improving the energy efficiency.

In this paper we adopt the spectrum-efficient fusion scheme of [23] and propose a new transmission strategy for saving energy in the report channel transmissions, without compromising the global decision performance. In our scheme, the simultaneous transmission technique proposed in [23] is combined with censoring [25] in the reporting phase, as follows: after local spectrum sensing, only the SUs that detect the presence of the PU signal are allowed to transmit the sensing information to the FC. The SUs that find no active PU signal do not send any information to the FC, decreasing the total energy consumption in the cognitive network. To reach the global decision the FC only needs to know how many SUs have reported their local decisions, which transforms the complex original decision rule of [23] into a simple threshold-based decision scheme. We derive expressions for computing the key performance metrics of the spectrum sensing (the probabilities of detection and false alarm), and these expressions are validated through computer simulations. An extensive analysis about the total energy consumption is also made for our fusion scheme, as well as for the one proposed in [23], allowing for a fair comparison between them in terms of energy efficiency. We also present a comparison between our fusion scheme and the censoring scheme proposed in [22], due to the similarity between them in one specific configuration of the scheme of [22].

The remaining of the paper is organized as follows: the system model proposed in [23] is discussed in Section 2. Section 3 is devoted to the proposed censored decision and energy efficient fusion system. The analysis of the energy efficiency is provided in Section 4. Our system is compared with a similar one in Section 5. Section 6 concludes the paper.

\section{Reference System}

In this section we first present the system model proposed in [23], which we hereafter refer to as the reference system, and give the expressions for its performance analysis. Then we discuss the accuracy of such expressions when compared with simulations. Subsequently, we discuss the energy efficiency of the reference system.

\subsection{Reference system model}

Basically, the whole process in the CSS scheme proposed in [23] can be divided into three phases: i) local spectrum sensing, ii) local decision transmission and iii) global decision at the FC. In the first phase, the activity of the PU is observed by each SU. The hypotheses $H_{1}$ and $H_{0}$ denote the PU signal presence and absence, respectively. The $k$ th SU forms its local decision as $m_{k}=1$ if $T>\xi$, and $m_{k}=0$ if $T<\xi$, where $T$ is the test statistic for the detection technique ${ }^{1}$ adopted by the SUs, and $\xi$ is a local decision threshold, which is determined according to the desired probabilities of detection and false alarm. In the second phase, the SUs simultaneously transmit their local decisions by converting $m_{k}$ into BPSK symbols that can be represented in baseband as $s_{k}=\left(2 m_{k}-1\right) \sqrt{E_{\mathrm{s}}}$, where $E_{\mathrm{s}}$ is the average energy per transmitted symbol, which is equal to average energy per bit $E_{\mathrm{b}}$. Therefore, the received signal sample at the fusion center is given by

\footnotetext{
${ }^{1}$ Common techniques that can be adopted by the SUs are the energy detection, the cyclostationary feature detection, the matched filter detection and the eigenvalue-based detection [3].
} 


$$
r=\sum_{k=0}^{M-1} h_{k} s_{k}+w
$$

where $M$ is the number of cooperating SUs in the cognitive network, $h_{k}$ is the gain of the report channel between the $k$ th SU and the FC, and $w$ is the additive white Gaussian noise (AWGN) at the FC receiver input, having zero mean, variance $\sigma_{w}^{2}$ and unilateral power spectral density $N_{0}=2 \sigma_{w}^{2}$ watts per hertz.

It is assumed in [23] that $h_{k}$ is known at the FC, which allows for the classification of the local decision vector $s=\left[s_{0}, s_{1}, \cdots, s_{M-1}\right]^{\mathrm{T}}$ as belonging to one of two sets, $D_{0}$ or $D_{1}$, respectively associated with the hypotheses $H_{0}$ and $H_{1}$, under the $K$-out-of- $M$ decision fusion rule. In this rule, the FC chooses $H_{1}$ when at least $K$ among $M$ secondary users declare an active primary user in the band of interest. According to the decision rule given in [23], the FC will choose $H_{1}$ if

$$
\sum_{s \in D_{1}} \exp \left[-\frac{\left(r-\sum_{k=0}^{M-1} h_{k} s_{k}\right)^{2}}{2 \sigma_{w}^{2}}\right] \geq \sum_{s \in D_{0}} \exp \left[-\frac{\left(r-\sum_{k=0}^{M-1} h_{k} s_{k}\right)^{2}}{2 \sigma_{w}^{2}}\right]
$$

and will choose $H_{0}$ otherwise.

If AWGN report channels are considered, the noiseless received signal samples $r=\sum_{k=0}^{M-1} h_{k} s_{k}$ follow a Binomial distribution with $M+1$ values that can be represented geometrically as points in a one-dimensional space. The points $\left\{(2 K-M) \sqrt{E_{\mathrm{b}}}, \cdots, M \sqrt{E_{\mathrm{b}}}\right\}$ belong to the set $D_{1}$, and the points $\left\{-M \sqrt{E_{\mathrm{b}}}, \cdots,(2 K-M-2) \sqrt{E_{\mathrm{b}}}\right\}$ belong to the set $D_{0}$.

Applying the max-log approximation $\ln \sum_{i} \exp \left(a_{i}\right) \simeq \max \left(a_{i}\right)$, it can be shown that the global probability of detection and false alarm at the FC can be calculated as [23-24]

$$
\begin{aligned}
P_{\mathrm{D}, \mathrm{FC}} & =\sum_{l=0}^{M} Q\left[(2 K-2 l-1) \sqrt{2 \gamma_{\mathrm{FC}}}\right] \times\left(\begin{array}{c}
M \\
l
\end{array}\right) P_{\mathrm{D}, \mathrm{SU}}^{l}\left(1-P_{\mathrm{D}, \mathrm{SU}}\right)^{M-l} \\
P_{\mathrm{FA}, \mathrm{FC}} & =\sum_{l=0}^{M} Q\left[(2 K-2 l-1) \sqrt{2 \gamma_{\mathrm{FC}}}\right] \times\left(\begin{array}{c}
M \\
l
\end{array}\right) P_{\mathrm{FA}, \mathrm{SU}}^{l}\left(1-P_{\mathrm{FA}, \mathrm{SU}}\right)^{M-l}
\end{aligned}
$$

where $Q(\cdot)$ is the Gaussian tail probability function, $P_{\mathrm{D}, \mathrm{SU}}$ and $P_{\mathrm{FA}, \mathrm{SU}}$ are the probabilities of detection and false alarm at the SUs and $\gamma_{\mathrm{FC}}=E_{\mathrm{s}} / N_{0}$ is the average SNR per bit at the FC.

As pointed out in [24], when the reporting channel gains are complex-valued and possibly time-varying, the decision regions become two-dimensional, with irregular and random shape, rendering the derivation of the probabilities of false alarm and detection a much more challenging task when compared to the case of real-valued gains. However, one must recall that the fusion scheme proposed in [23] assumes that the channel gains are known at the FC, which means that the signal levels can be differentiated for the proper operation of the 
$K$-out-of- $M$ rule. In terms of performance, it can happen, for instance, that two closely-nearby signal points will belong to different hypotheses, no matter how large the SNR is. In this case, the instantaneous performance is expected to be severely degraded, as also happens, for instance, in a deep fading condition caused by destructive interference.

\subsection{On the adherence between theoretical and actual results for the reference system}

The main conclusion in [23] is that its resource-efficient scheme is capable of achieving the target values of $P_{\mathrm{D}, \mathrm{FC}}=0.9$ and $P_{\mathrm{FA}, \mathrm{FC}}=0.1$, as does a scheme that applies orthogonal transmissions over the report channels. However, this is accomplished only when the SNR in the report channel $\left(\gamma_{\mathrm{FC}}\right)$ is relatively high, for example above around $6 \mathrm{~dB}$ for AWGN report channels [24]. Moreover, the max-log approximation used to derive (3) and (4) from (2) is accurate only in specific cases: i) when $K=\lceil M / 2\rceil$ in the $K$-out-of- $M$ rule, or ii) when the values of $\gamma_{\mathrm{FC}}$ is high, normally above $6 \mathrm{~dB}$. As a consequence, theoretical and actual performances of the scheme proposed in [23] only match in special cases, as reported in [24].

The mismatch between the theoretical and the actual performances of the scheme proposed in [23] can be justified firstly by the use of the max-log approximation in the derivation of the expressions for computing the probabilities of false alarm and detection in [23], that is, the logarithm of the sum of exponentials in (2) was replaced by the max function. However, the max function close approximates the log of the sum of exponentials only when the exponents are quite different, meaning that the sum will be governed mainly by the largest one. When $\gamma_{\mathrm{FC}}$ is high, the differences among the exponents in (2) are large, meaning that the max-log approximation is accurate. When $\gamma_{\mathrm{FC}}$ is low, the differences among the exponents in (2) tend to become small, penalizing the accuracy of the approximation. In the specific case of $K=\lceil M / 2\rceil$ in the $K$-out-of- $M$ rule, no matter the value of $\gamma_{\mathrm{FC}}$, any inaccuracy of the max-log approximation penalizes equally both sides of (2), since the number of terms in both summations is the same.

The above considerations can be more clearly addressed by means of receiver operating characteristic (ROC) curves, which show the probability of detection versus the probability of false alarm as a decision threshold $\xi$ at the SU is varied. No specific detection technique at the SUs was called in [23], since it does not influence the operation of the decision fusion strategy. Furthermore, it was indeed not necessary to define any detection technique in [23], because the performance results report the global probabilities of detection and false alarm as a function of $\gamma_{\mathrm{FC}}$. Nevertheless, hereafter we consider that the $M$ cooperating SUs perform energy detection (ED). In this case, a local test statistic is $T=(1 / \sigma) \sum_{n=0}^{N-1}|x[n]|^{2}$, where $\sigma$ is the noise variance at the input of each SU receiver, $N$ is number of samples collected by each $\mathrm{SU}$, and $x[n]$ is the received signal sample collected by an SU in the $n$th discrete-time instant. Notice that, by defining the above test statistic independent of the specific SU, we are implicitly assuming that the noise variance and the sensing performances of all SUs are the same. Then, the detection and false alarm probabilities at the SUs can be respectively computed as [26]

$$
P_{\mathrm{D}, \mathrm{SU}}=P\left[T \geq \xi \mid H_{1}\right] \simeq Q\left(\frac{\xi-N\left(\sigma^{2}+\sigma_{x}^{2}\right)}{\sqrt{2 N\left(\sigma^{2}+\sigma_{x}^{2}\right)^{2}}}\right)
$$




$$
P_{\mathrm{FA}, \mathrm{SU}}=P\left[T \geq \xi \mid H_{0}\right] \simeq Q\left(\frac{\xi-N \sigma^{2}}{\sqrt{2 N \sigma^{4}}}\right)
$$

where $\sigma_{x}^{2}$ is the variance of the PU signal. The average signal-to-noise ratio for the links between the PU and the SUs is simply $\gamma_{\mathrm{SU}}=\sigma_{x} / \sigma$.

From Fig. 1 it can be seen that theoretical and simulated performance results for the reference system of [23] match for higher values of $\gamma_{\mathrm{FC}}$, being considerably different in lower values of $\gamma_{\mathrm{FC}}$. To plot these results, we have arbitrarily assumed $N=100, M=5, K=1$ (left) and $K=M=5$ (right), $\gamma_{\mathrm{SU}}=-5 \mathrm{~dB}$ and $\gamma_{\mathrm{FC}}=0 \mathrm{~dB}$ and $8 \mathrm{~dB}$. The results for $K=\lceil M / 2\rceil=3$ were omitted since, in this specific case, the theoretical and simulation results always agree. Notice that these values of $K$ configure the well-known decision fusion rules OR, AND, and majority-voting, respectively. Each value on the simulated ROC curves was obtained from 500,000 Monte Carlo events. Each event corresponds to computing $P_{\mathrm{D}, \mathrm{SU}}$ and $P_{\mathrm{FA}, \mathrm{SU}}$ from (5) and (6), respectively, for a given decision threshold $\xi$ and signal-to-noise ratio $\gamma_{\mathrm{SU}}$. The probability $P_{\mathrm{D}, \mathrm{SU}}$ is set as the probability of success in the Binomial distribution for generating a set of SUs decisions when the PU signal is on. Similarly, $P_{\mathrm{FA}, \mathrm{SU}}$ governs the Binomial distribution of the SUs decisions when the PU signal is off. The SUs decisions are then sent to the FC through the report channel using BPSK signaling. The final decision at the FC are used for computing false alarm (if the PU is off) and detection (if the PU is on) rates, which are the estimates of the associated probabilities. Repeating the above procedure, by varying the decision threshold at the SUs, the ROC curves are traced out.
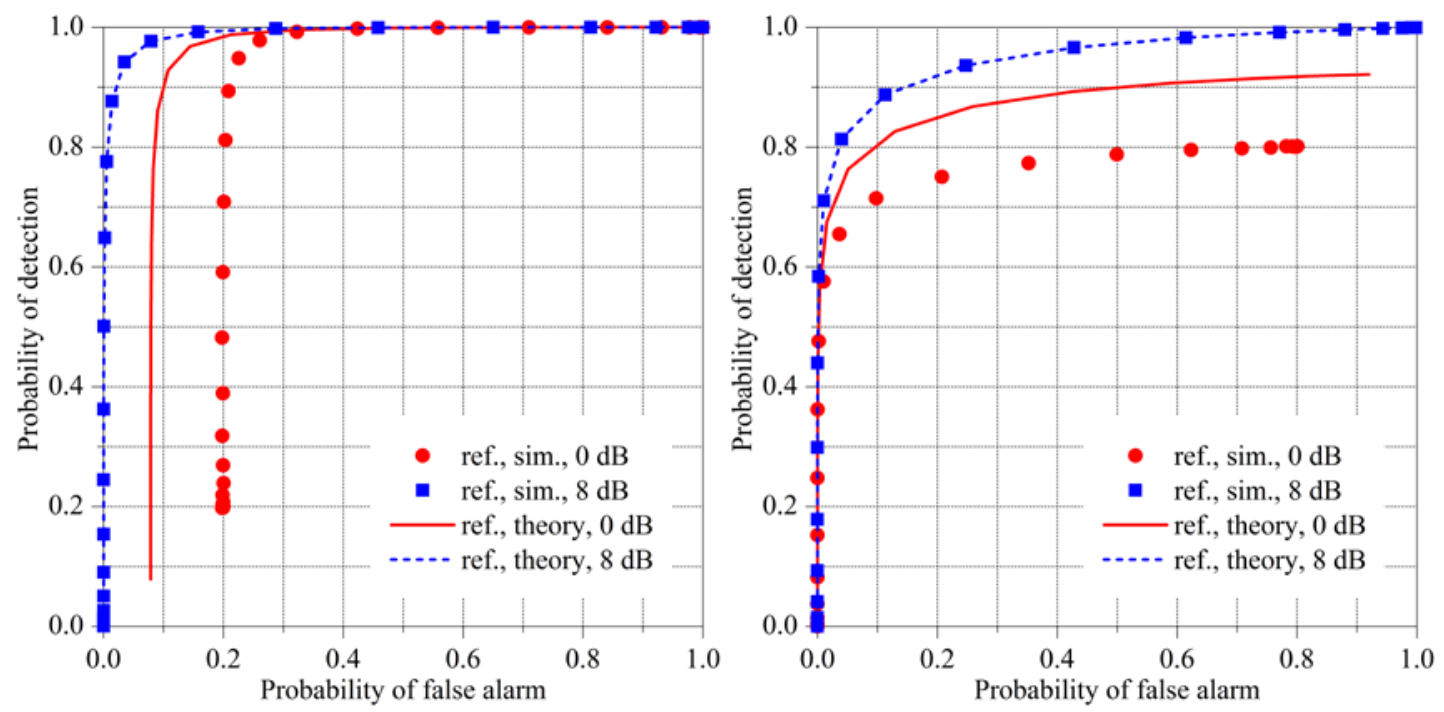

Fig. 1. Differences among theoretical and simulated ROC results for the reference system proposed in [23]. $K=1$ left, $K=5$ right.

\subsection{On the energy consumption of the reference system}

In this section we make an analysis about the energy consumption in the CSS network under the scheme proposed in [23]. 
As described in Section 2.1, the CSS of [23] is based on the simultaneous transmissions of all secondary users' decisions to the FC. So, the total energy consumption in the cognitive network per sensing round can be formulated as [20]

$$
E_{\text {ref }}=M E_{\mathrm{LS}}+M E_{\mathrm{R}}+P_{\text {free }} E_{\mathrm{T}}
$$

where $E_{\mathrm{LS}}, E_{\mathrm{R}}$ and $E_{\mathrm{T}}$ are the energy consumed by a single SU due to local sensing, reporting of the local decisions to the FC and opportunistic data transmission, respectively. The first term in (7) is constant and exists in all sensing rounds. Using lower values for $M$ we can reduce the contribution of this term, but this will penalize the performance of the CSS. The second term in (7) also increases with $M$, and represents a significant contribution in the total energy consumption. Since the SUs transmit their local decisions in all reporting phases via BPSK symbols, the energy consumption $M E_{\mathrm{R}}$ is always equal to $M E_{\mathrm{b}}$. The last term depends on the probability that the FC decides in favor of a free channel, which is given by $P_{\text {free }}=P_{H_{1}}\left(1-P_{\mathrm{D}, \mathrm{FC}}\right)+P_{H_{0}}\left(1-P_{\mathrm{FA}, \mathrm{FC}}\right)$, where $P_{\mathrm{H}_{1}}$ and $P_{H_{0}}=\left(1-P_{H_{1}}\right)$ are, respectively, the probabilities that the PU transmitter is on and off, and $P_{\mathrm{D}, \mathrm{FC}}$ and $P_{\mathrm{FA}, \mathrm{FC}}$ are computed from (3) and (4), respectively.

The energy efficient fusion system described in the next section aims at reducing the total energy consumption based on a censored local decision reporting strategy, which acts by reducing the second term in (7).

\section{Proposed Energy Efficient System}

As in the case of the reference system, the whole process in the censored CSS scheme proposed here can be divided into three phases: i) local spectrum sensing, ii) reporting of local decisions and iii) global decision at the FC. The differences between our scheme and the reference reside in phases ii and iii.

\subsection{Proposed system model}

To achieve the same bandwidth efficiency of the reference system suggested in [23], the new energy efficient CSS scheme also adopts the simultaneous transmission of local decisions to the FC over the same frequency band. However, differently of [23], only the SUs that detect the presence of a PU signal are allowed to report their decisions to the FC. The remaining SUs must stay silent. Therefore, the received signal sample at the FC can be expressed as

$$
r_{\text {new }}=\sum_{k=0}^{M^{*}} h_{k} s_{k}+w
$$

where, $M^{*} \in[0, M]$ is an integer that represents the number of SUs that have reported their local decision or, in other words, it is the number of SUs with local decision $m_{k}=1$.

Notice that during the reporting phase, the transmission of information occurs only if a detection or a false alarm event occurs. The SUs' transmitters remain turned-off otherwise. Therefore, the average energy per bit is computed conditioned on transmission events. So, as in the case of the reference system, $E_{\mathrm{b}}=E_{\mathrm{s}}$ and, for the same $\gamma_{\mathrm{FC}}$ in new and the reference 
schemes, a fair comparison in terms of the global decision performances among these two techniques can be made.

Again assuming AWGN report channels, the noiseless received signal sample in this new scheme also follows a Binomial distribution with $M+1$ values, now represented as depicted in Fig. 2.

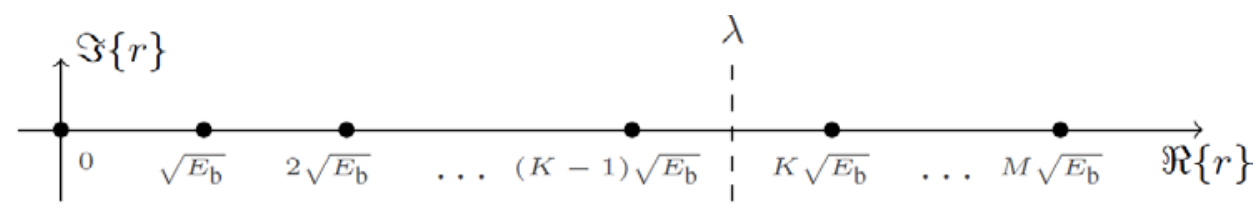

Fig. 2. Noiseless received symbols at the FC for the new fusion scheme.

Each point in Fig. 2 is associated to an event in which $J$ SUs send the local decision, $J=0,1, \cdots, M$. Under the $K$-out-of- $M$ rule, the points $\left\{0, \sqrt{E_{\mathrm{b}}}, \cdots,(K-1) \sqrt{E_{\mathrm{b}}}\right\}$ correspond to the choice of $H_{0}$, and the points $\left\{K \sqrt{E_{\mathrm{b}}}, \cdots, M \sqrt{E_{\mathrm{b}}}\right\}$ correspond to the choice of $H_{1}$. Therefore, the FC will choose $H_{1}$ if $r_{\text {new }} \geq \lambda$ and $H_{0}$ otherwise. Notice that this threshold-based rule is by far less complex than (2), which is the decision rule adopted in the reference system. On the basis of Fig. 2, the global probabilities of detection and false alarm for the new energy efficient scheme can be derived in a manner similar to the one adopted in [24], leading to

$$
\begin{gathered}
P_{\mathrm{D}, \mathrm{FC}}=P\left[r_{\mathrm{new}} \geq \lambda \mid H_{1}\right]=\sum_{l=0}^{M} Q\left[\lambda-l \sqrt{2 \gamma_{\mathrm{FC}}}\right] \times\left(\begin{array}{c}
M \\
l
\end{array}\right) P_{\mathrm{D}, \mathrm{SU}}^{l}\left(1-P_{\mathrm{D}, \mathrm{SU}}\right)^{M-l} \\
P_{\mathrm{FA}, \mathrm{FC}}=P\left[r_{\mathrm{new}} \geq \lambda \mid H_{0}\right]=\sum_{l=0}^{M} Q\left[\lambda-l \sqrt{2 \gamma_{\mathrm{FC}}}\right] \times\left(\begin{array}{c}
M \\
l
\end{array}\right) P_{\mathrm{FA}, \mathrm{SU}}^{l}\left(1-P_{\mathrm{FA}, \mathrm{SU}}\right)^{M-l}
\end{gathered}
$$

Without loss of generality, we assume unitary average bit energy, i.e. $E_{\mathrm{b}}=E_{\mathrm{s}}$. Then, from Fig. 2, the decision threshold $\lambda \in[K-1, K]$. The correct choice of $\lambda$ in this interval can lead to a better system performance in terms of ROC. In other words, we can find the value of $\lambda$, restricted to $K-1 \leq \lambda \leq K$, that minimizes the probability of making a wrong decision at the $\mathrm{FC}$, that is

$$
\lambda_{\mathrm{opt}}=\underset{\lambda \in[K-1, K]}{\arg \min }\left\{P_{\mathrm{e}, \mathrm{FC}}(\lambda)\right\}
$$

where $P_{\mathrm{e}, \mathrm{FC}}(\lambda)$ is the probability of occurrence of a false alarm or a missed detection as a function of $\lambda$. This probability is given by

$$
P_{\mathrm{e}, \mathrm{FC}}(\lambda)=P_{H_{0}} P_{\mathrm{FA}, \mathrm{FC}}(\lambda)+P_{\mathrm{H}_{1}}\left\{1-P_{\mathrm{D}, \mathrm{FC}}(\lambda)\right\}
$$

where $P_{\mathrm{D}, \mathrm{FC}}(\lambda)$ and $P_{\mathrm{FA}, \mathrm{FC}}(\lambda)$ are computed from (9) and (10), respectively. However, it is not reasonable to assume that the values of $P_{\mathrm{D}, \mathrm{SU}}$ and $P_{\mathrm{FA}, \mathrm{SU}}$ required to compute $P_{\mathrm{D}, \mathrm{FC}}(\lambda)$ and $P_{\mathrm{FA}, \mathrm{FC}}(\lambda)$ are known at the FC. Then, to find $\lambda_{\mathrm{opt}}$ here we consider that the local 
spectrum sensing at the SUs are designed to achieve target values of false alarm and detection probabilities for an error-free report channel condition. We refer to these target values as $P_{\mathrm{FA}, \mathrm{SU}}^{(\mathrm{T})}$ and $P_{\mathrm{D}, \mathrm{SU}}^{(\mathrm{T})}$, respectively.

We have adopted two rules to determine $P_{\mathrm{FA}, \mathrm{SU}}^{(\mathrm{T})}$ and $P_{\mathrm{D}, \mathrm{SU}}^{(\mathrm{T})}$. In the first rule, the target performance metrics at the $\mathrm{FC}, P_{\mathrm{FA}, \mathrm{FC}}^{(\mathrm{T})}$ and $P_{\mathrm{D}, \mathrm{FC}}^{(\mathrm{T})}$, are chosen to meet performance specifications, for example to comply with standard requirements. In this case, $P_{\mathrm{FA}, \mathrm{SU}}^{(\mathrm{T})}$ and $P_{\mathrm{D}, \mathrm{SU}}^{(\mathrm{T})}$ are computed as the solutions of the following equations [27]

$$
\begin{gathered}
P_{\mathrm{D}, \mathrm{FC}}^{(\mathrm{T})}=\sum_{l=K}^{M}\left(\begin{array}{c}
M \\
l
\end{array}\right) P_{\mathrm{D}, \mathrm{SU}}^{(\mathrm{T})} l\left(1-P_{\mathrm{D}, \mathrm{SU}}^{(\mathrm{T})}\right)^{M-l} \\
P_{\mathrm{FA}, \mathrm{FC}}^{(\mathrm{T})}=\sum_{l=K}^{M}\left(\begin{array}{c}
M \\
l
\end{array}\right) P_{\mathrm{FA}, \mathrm{SU}}^{(\mathrm{T})} l\left(1-P_{\mathrm{FA}, \mathrm{SU}}^{(\mathrm{T})}\right)^{M-l}
\end{gathered}
$$

In the second rule for determining $P_{\mathrm{FA}, \mathrm{SU}}^{(\mathrm{T})}$ and $P_{\mathrm{D}, \mathrm{SU}}^{(\mathrm{T})}$, we have used the fact that the minimum decision error probability at the FC happens when the decision error probability at the SUs also attains its minimum. Then, we assume that $P_{\mathrm{FA}, \mathrm{SU}}^{(\mathrm{T})}$ and $P_{\mathrm{D}, \mathrm{SU}}^{(\mathrm{T})}$ are computed from (5) and (6) for a given set of system parameters and for a target SU decision threshold $\xi^{(\mathrm{T})}$ that satisfies

$$
\xi^{(\mathrm{T})}=\underset{\xi}{\arg \min }\left\{P_{\mathrm{e}, \mathrm{SU}}(\xi)\right\}=\underset{\xi}{\arg \min }\left\{P_{\mathrm{H}_{0}} P_{\mathrm{FA}, \mathrm{SU}}(\xi)+P_{\mathrm{H}_{1}}\left[1-P_{\mathrm{D}, \mathrm{SU}}(\xi)\right]\right\}
$$

where the functions $P_{\mathrm{FA}, \mathrm{SU}}(\xi)$ and $P_{\mathrm{D}, \mathrm{SU}}(\xi)$ are also defined from (5) and (6).

Even assuming target probabilities at the SUs, it is not trivial to find the value of $\lambda \in[K-1, K]$ that minimizes the error probability at the FC: to solve (11) analytically, one would have to operate with a summation of exponentials that are functions of $\lambda$ and whose terms are weighted by binomial probabilities. These exponentials arise from the derivative of the Gaussian $Q$ function present in (9) and (10). For this reason we have solved (11) numerically.

\subsection{Performance results for the proposed system}

The accuracy of our expressions is evidenced in Fig. 3 and Fig. 4, where the global probabilities of detection and false alarm for the new and the reference schemes are shown for $K=1$ (left) and $K=3$ (right). To plot Fig. 3, the target metrics chosen according to the first rule was $P_{\mathrm{FA}, \mathrm{FC}}^{(\mathrm{T})}=0.1$ and $P_{\mathrm{D}, \mathrm{FC}}^{(\mathrm{T})}=0.9$, yielding, from the solution of (13) and (14), $P_{\mathrm{FA}, \mathrm{SU}}^{(\mathrm{T})}=0.0209$ and $P_{\mathrm{D}, \mathrm{SU}}^{(\mathrm{T})}=0.3690$ for $K=1$ with $\lambda_{\mathrm{opt}}=0.9649$ for $\gamma_{\mathrm{FC}}=0 \mathrm{~dB}$ and $\lambda_{\text {opt }}=0.6112$ for $\gamma_{\mathrm{FC}}=8 \mathrm{~dB}$; and $P_{\mathrm{FA}, \mathrm{SU}}^{(\mathrm{T})}=0.2466$ and $P_{\mathrm{D}, \mathrm{SU}}^{(\mathrm{T})}=0.7534$ for $K=3$ with $\lambda_{\text {opt }}=2.5$ for both values of $\gamma_{\mathrm{FC}}$. To plot Fig. 4, the target metrics computed according to the second rule was $P_{\mathrm{FA}, \mathrm{SU}}^{(\mathrm{T})}=0.1305$ and $P_{\mathrm{D}, \mathrm{SU}}^{(\mathrm{T})}=0.8009$ for any value of $K$, while $\lambda_{\text {opt }}=1$ if 
$K=1$ and $\lambda_{\mathrm{opt}}=4$ if $K=5$ for both values of $\gamma_{\mathrm{FC}}$, lastly if $K=3, \lambda_{\mathrm{opt}}=2.2758$ for $\gamma_{\mathrm{FC}}=0 \mathrm{~dB}$ and $\lambda_{\mathrm{opt}}=2.4117$ for $\gamma_{\mathrm{FC}}=8 \mathrm{~dB}$. We have adopted $P_{\mathrm{H}_{0}}=P_{\mathrm{H}_{1}}=0.5$ in order to simulate the most difficult situation (maximal entropy of the primary user activity).
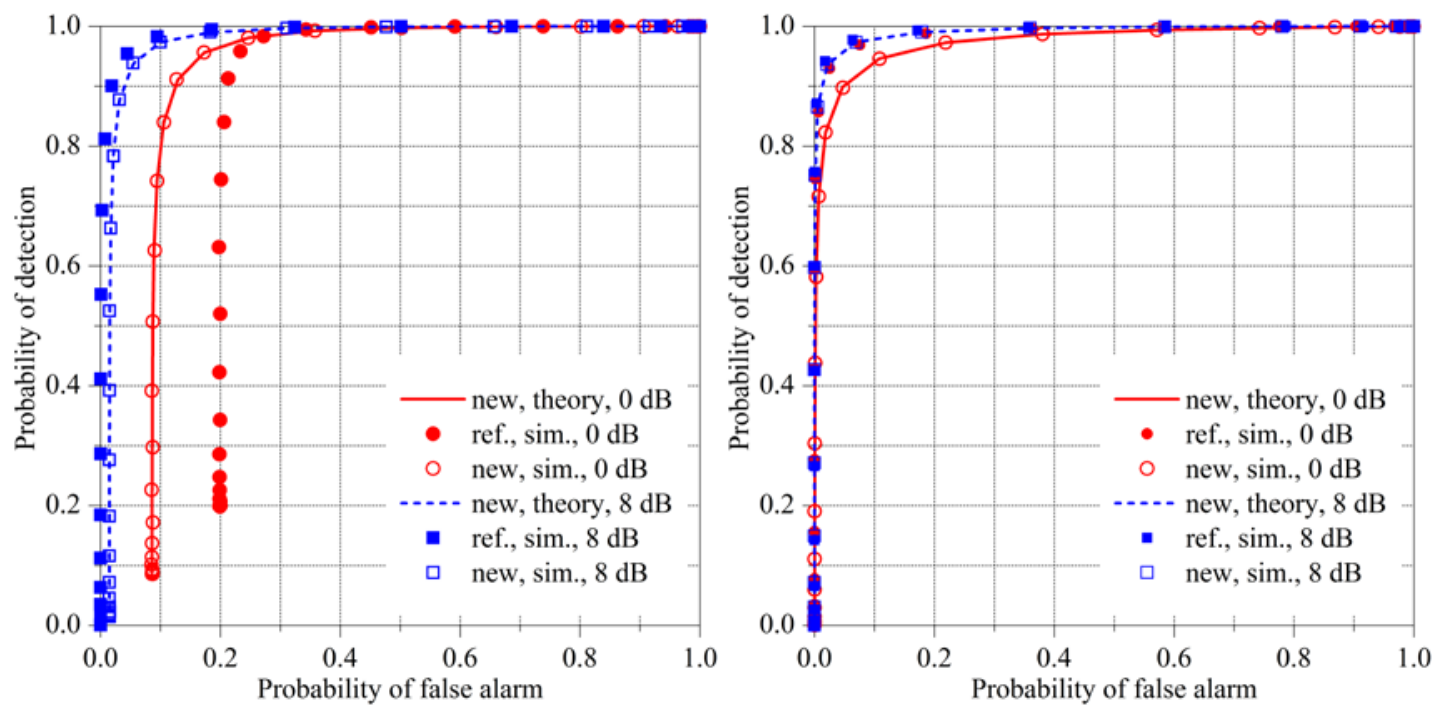

Fig. 3. Global probabilities of detection and false alarm for the new and the reference schemes. $K=1$ left, $K=3$ right. First rule adopted for determining target metrics.
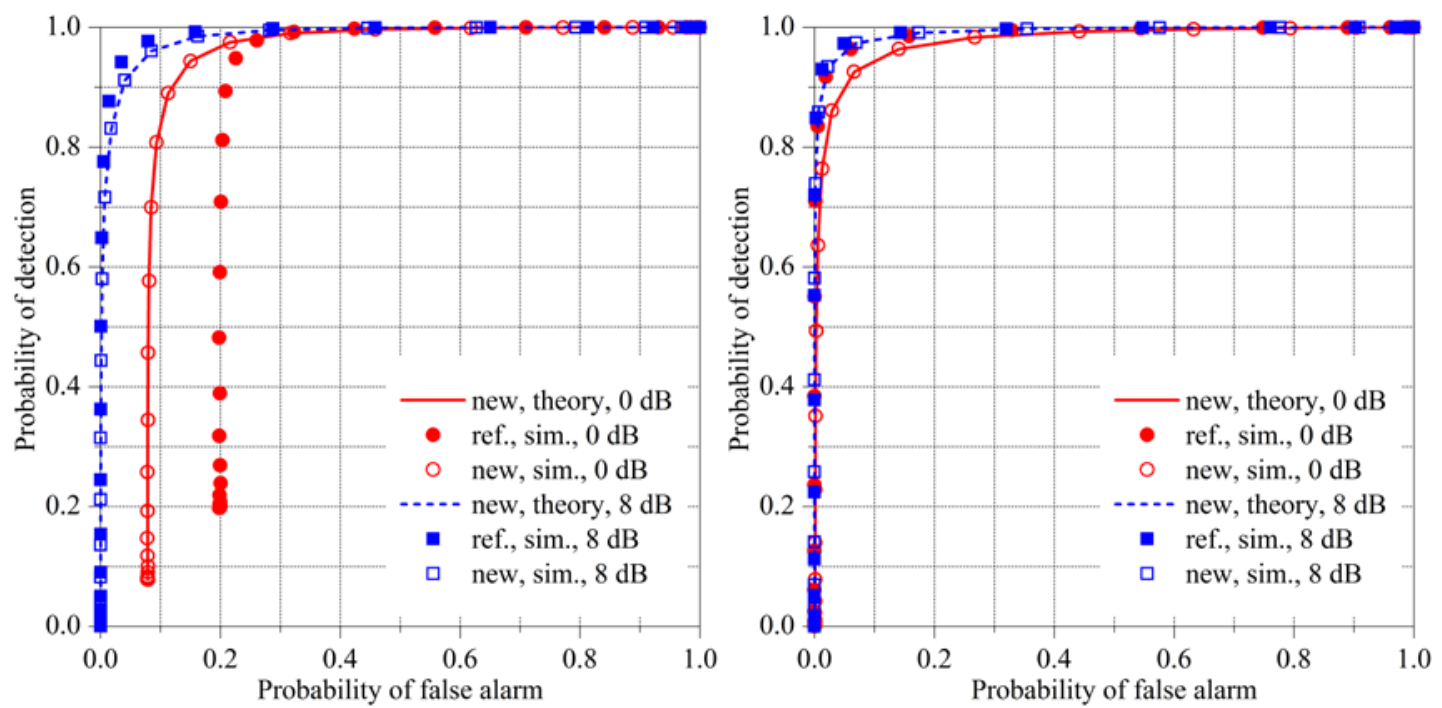

Fig. 4. Global probabilities of detection and false alarm for the new and the reference schemes. $K=1$ left, $K=3$ right. Second rule adopted for determining target metrics.

Notice that the two rules for choosing the decision threshold lead to very similar ROC performances of the proposed scheme, mainly for $K=3$. Notice that the graphs on the right of Figs. 3 and $\mathbf{4}$ present almost equal ROC performances. When $K=1$, it can be observed a small advantage of the second rule. For both $\gamma_{\mathrm{FC}}=0 \mathrm{~dB}$ and $\gamma_{\mathrm{FC}}=8 \mathrm{~dB}$, the first rule leads to a minimum value of the global false alarm slightly greater than the second rule. Then, it is 
possible to conclude that these two rules can be indistinguishably used in the proposed scheme, without significant implications in performance.

It can be also observed from these figures that, when comparing our scheme (new) with the one proposed in [23] (reference), the new scheme overcomes the reference for $\gamma_{\mathrm{FC}}=0 \mathrm{~dB}$ and the performances are approximately equal when $\gamma_{\mathrm{FC}}=8 \mathrm{~dB}$. When $K=3$ the performances of both schemes are practically the same for all values of $\gamma_{\mathrm{FC}}$.

We conclude that the new scheme can save energy during the reporting phase without compromising the global decision performance, improving this performance in some cases. This improvement can be explored for reducing the second term of (7), saving even more energy. More details regarding this aspect are given in the next subsection.

It is worth mentioning that the performance results of the reference system as shown in Fig. 3 and Fig. $\mathbf{4}$ are from simulations because the theoretical results from [23] do not match the simulations for $\gamma_{\mathrm{FC}}=0 \mathrm{~dB}$; see Section 2.2.

As for the previous ROCs, each value on the curves from simulations in Fig. $\mathbf{3}$ and Fig. 4 was obtained from 500,000 Monte Carlo events. The difference from the previous setup in each event, when the new system is considered, is that the SU's decisions are sent to the FC using the proposed censoring technique, and the final decision at the FC is arrived at from the simple threshold-based decision rule. The received SNR at the SUs was arbitrarily set to $\gamma_{\mathrm{SU}}=-5 \mathrm{~dB}$.

\subsection{On the energy consumption of the proposed system}

Similarly to what we have done regarding the reference system, we can model the total energy consumption per sensing round for the new system as

$$
E_{\text {new }}=M E_{\mathrm{LS}}+\overline{M^{*}} E_{\mathrm{R}}+P_{\text {free }} E_{\mathrm{T}}
$$

recalling that $E_{\mathrm{LS}}, E_{\mathrm{R}}$ and $E_{\mathrm{T}}$ are the energy consumed by a single $\mathrm{SU}$ due to local sensing, reporting of the local decisions to the FC and opportunistic data transmission, respectively, $M$ is the number of cooperating SUs, $\overline{M^{*}}$ is the average number of SUs with local decision $m_{k}=1$ and $P_{\text {free }}$ is the probability that the FC decides in favor of a free channel. The first term of (16) is constant and has the same value both in (7) and (16). The second term in the reference system is also constant and equal to $M E_{\mathrm{b}}$. However, in the proposed scheme this term depends on $\overline{M^{*}}=M P\left[m_{k}=1\right]$, where $P\left[m_{k}=1\right]=P_{H_{0}} P_{\mathrm{FA}, \mathrm{SU}}+P_{H_{1}} P_{\mathrm{D}, \mathrm{SU}}$. Therefore, the energy consumption in the reporting phase for the new scheme is $\overline{M^{*}} E_{\mathrm{R}}=M E_{\mathrm{b}}\left(P_{\mathrm{H}_{0}} P_{\mathrm{FA}, \mathrm{SU}}+P_{H_{1}} P_{\mathrm{D}, \mathrm{SU}}\right)$.

The maximum value of energy consumption in the reporting phase of the new scheme is equal to $M E_{\mathrm{b}}$, a value that is equal to the corresponding one in the reference model and, when all SUs decide by $m_{k}=0$, the energy consumption in the reporting phase is zero. However, on the average, the energy consumption in the new scheme will be lower than in the reference scheme. The last term also depends on $P_{\text {free }}$, as in the reference model. Recall that the energy consumption of the new scheme is reduced compared to the reference scheme due to a smaller second term of (16). 


\section{Energy Efficiency Analysis}

We present the energy efficiency analysis using two approaches. The first is made considering a scenario in which the global decision performances of the reference and new schemes are equal to one another. The second considers the possibility of different performances. For both approaches we have arbitrarily adopted the second rule for determining the target metrics, since this rule has produced approximately the same results achieved with the first one; refer to Section 3 for more details about these rules.

For the first analysis we consider $M=5, K=3, \gamma_{\mathrm{FC}}=8 \mathrm{~dB}$ and $\gamma_{\mathrm{SU}}=-5 \mathrm{~dB}$. With these parameters, the global decision performances are approximately the same for the new and the reference schemes, as shown in Fig. 3 and Fig. 4; see Section 3. Therefore, the values of the third term in (7) and (16) are approximately equal. As the first term in these two equations always have equal values, this first analysis will show a comparison in terms of energy consumption mainly due to the reporting phase.

In Fig. 5 we present the energy consumption per sensing round for the new and the reference schemes, as respectively obtained from (7) and (16). The results show that the new scheme achieves significant reduction in the energy consumption when compared with the reference. For obtaining these results, the average energy per bit was set to $E_{\mathrm{b}}=E_{\mathrm{T}}=1$ joule, and the energy spent during the local sensing period was not taken into account, since its value is the same in both systems.

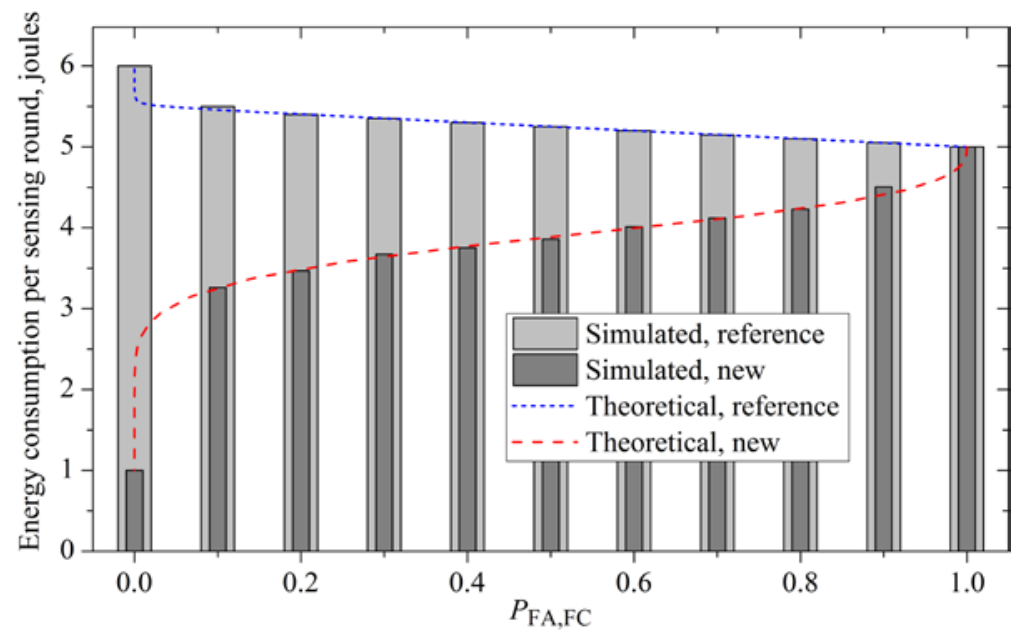

Fig. 5. Energy consumption per sensing round for the new and the reference schemes.

Notice in Fig. 3 and Fig. 4 that, for the parameters under analysis, $P_{\mathrm{FA}, \mathrm{FC}}=0$ implies $P_{\mathrm{D}, \mathrm{FC}}=0$. Therefore, for the reference system, in (7) $P_{\text {free }}=1$ and a secondary user transmits opportunistically with $E_{\mathrm{T}}=1$ joule. Since in the reporting phase $M$ BPSK symbols with energy $E_{\mathrm{b}}=1$ are transmitted, the energy consumption per sensing round (disregarding the consumption in the local sensing period) will be equal to $5 E_{\mathrm{b}}+1 E_{\mathrm{T}}=6$ joules, as show in Fig. 5. Under the same condition in the new scheme, $P_{\text {free }}$ is also unitary, so $P_{\text {free }} E_{\mathrm{T}}=1$. However, in the reporting phase there is no transmission of local decision, once $P_{\mathrm{FA}, \mathrm{FC}}=0$ was achieved by a condition in which $P_{\mathrm{FA}, \mathrm{SU}}=P_{\mathrm{D}, \mathrm{SU}}=0$. Therefore, for the new scheme the energy 
consumption per sensing round will be $0 E_{\mathrm{b}}+1 E_{\mathrm{T}}=1$ joule, as can also be observed in Fig. 5 . If $P_{\mathrm{FA}, \mathrm{SU}}$ increases, the total energy consumption in the reference system decreases due to a decrease in $P_{\text {free }}$. However, in the new scheme the total energy consumption increases, mainly due to an increase in the probability of a local detection in each SU, $P\left[m_{k}=1\right]$. Lastly, $P_{\mathrm{FA}, \mathrm{FC}}=1$ implies $P_{\mathrm{D}, \mathrm{FC}}=1$ (see Fig. 3 and Fig. 4) and, therefore, $P_{\text {free }}=0$, that is, no SU will transmit opportunistically. Thus, in the reference and in the new schemes, the difference in the total energy consumption will be the just the energy spent during the reporting phase. For the reference scheme, the reporting task always consumes $M E_{\mathrm{b}}=5$ joules. For the new scheme, $P_{\mathrm{FA}, \mathrm{FC}}=1$ was achieved by a condition where $P_{\mathrm{FA}, \mathrm{SU}}=P_{\mathrm{D}, \mathrm{SU}}=1$. Therefore, in this condition the energy spent during the reporting phase is $\overline{M^{*}} E_{\mathrm{R}}=M E_{\mathrm{b}}\left(P_{H_{0}} P_{\mathrm{FA}, \mathrm{SU}}+P_{\mathrm{H}_{1}} P_{\mathrm{D}, \mathrm{SU}}\right)=M E_{\mathrm{b}}=5$ joules, as shown in Fig. 5 . It is important to notice that expression (16) produces results in perfect agreement with the simulated results for any value of $\gamma_{\mathrm{FC}}$. On the other hand, expression (7) produces results in good agreement with simulations only for $\gamma_{\mathrm{FC}} \geq 6 \mathrm{~dB}$, approximately.

A second analysis is made by considering that the global decision performances are not necessarily the same for both systems. In this case, the energetic cost depends on the operating points of the reference and the proposed schemes. In other words, their global decision performances can influence the energy consumption, making a fair comparison between them difficult. Another metric can be incorporated into this comparison, accounting for the correct identification of the unused spectrum, which promotes the opportunistic data transmissions. This metric is the achievable throughput [18]

$$
D=P_{H_{0}}\left(1-P_{\mathrm{FA}, \mathrm{FC}}\right) R T_{t}
$$

in which $P_{\mathrm{H}_{0}}\left(1-P_{\mathrm{FA}, \mathrm{FC}}\right)$ is the probability of correct identification of the unused spectrum, $R$ is the data rate of the SU and $T_{t}$ is the time interval in which one of the SUs is scheduled for opportunistic data transmission. The energy efficiency, measured in bits per joule, is defined as the ratio between the achievable throughput and the total consumed energy, that is,

$$
\eta=D / E
$$

Fig. 6 shows the energy efficiency in both schemes, normalized by $R T_{t}$. The system parameters adopted were $M=5, \gamma_{\mathrm{SU}}=-5 \mathrm{~dB}$ and $\gamma_{\mathrm{FC}}=0 \mathrm{~dB}$ and $8 \mathrm{~dB}$. These results show that the new scheme is more efficient than the reference scheme in all analyzed conditions. Notice also that, if it is assumed a global $P_{\mathrm{FA}, \mathrm{FC}}=0.1, \gamma_{\mathrm{FC}}=0 \mathrm{~dB}$ and $K=1$, the energy efficiencies will be $\eta_{\text {new }}=0.25\left(R T_{t}\right)$ and $\eta_{\text {ref }}=0.08\left(R T_{t}\right)$, meaning a gain of about $212.5 \%$ in energy efficiency in favor of the proposed scheme. Similar behaviors were observed for other values of $M, K$ and $\gamma_{\mathrm{FC}}$, but with different efficiency gains.

It is informative to mention that the agreement between the theoretical and simulated energy efficiencies for the reference system is good only for high values of $\gamma_{\mathrm{FC}}$. Inaccuracies in the low $\gamma_{\mathrm{FC}}$ regime are inherited from the inaccuracy of the expressions for computing $P_{\mathrm{D}, \mathrm{FC}}$ and $P_{\mathrm{FA}, \mathrm{FC}}$ for the reference system in this regime. Last, but not least, it is important to notice that 
a higher energy efficiency can be obtained in our scheme not only from the reduction in the second term of (16), but also when the new scheme outperforms the reference one in terms of ROC, increasing the achievable throughput.

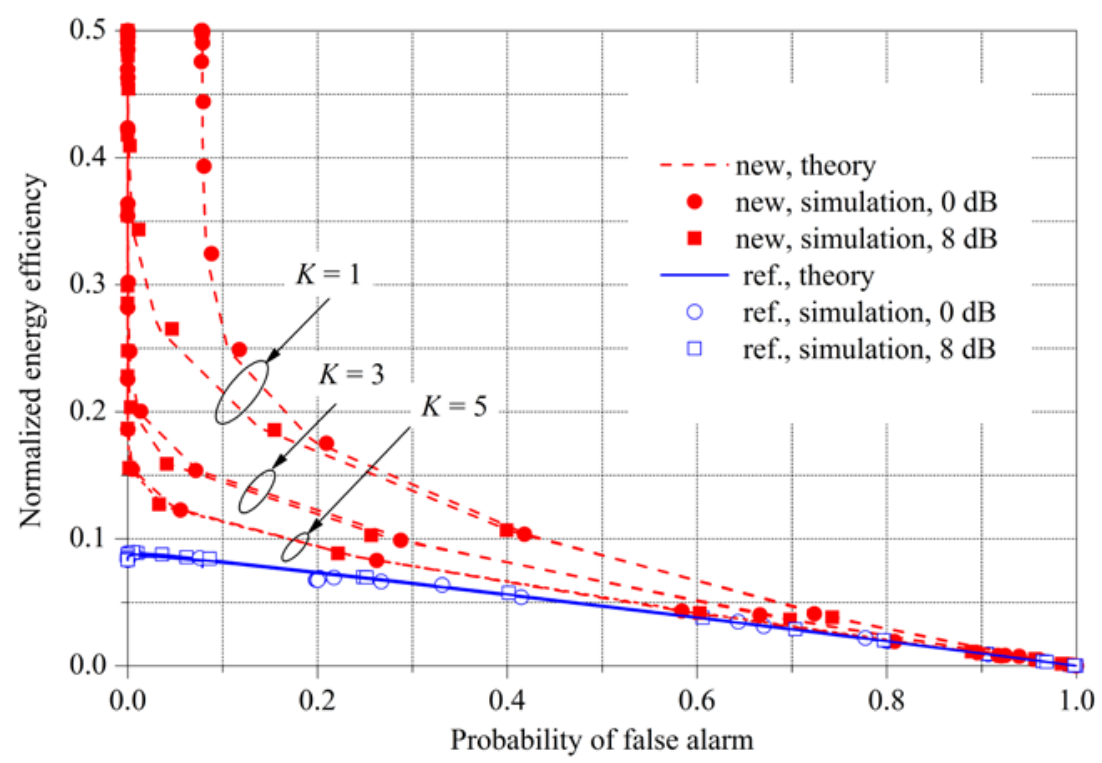

Fig. 6. Energy efficiencies of the reference and the new schemes.

From Fig. 6 it is also possible to notice that $\eta_{\text {new }}$ is more prone to variations with variations of the system parameters than $\eta_{\text {ref }}$. However, in all analyzed scenarios the energy efficiency of the new scheme is larger than the one achieved with the reference scheme. It is also important to notice that, for the new scheme, when $\gamma_{\mathrm{FC}}$ increases the energy efficiency decreases. This behavior is more pronounced when $K=1$ and $P_{\mathrm{FA}, \mathrm{FC}} \leq 0.2$ : analyzing this specific case, assuming $P_{\mathrm{FA}, \mathrm{FC}}=0.1$, one can observe in Fig. 4 that the global detection probability is higher in the case when $\gamma_{\mathrm{FC}}=8 \mathrm{~dB}$. Inverting (9) it is possible to notice that higher values of $P_{\mathrm{D}, \mathrm{SU}}$ are also achieved when $\gamma_{\mathrm{FC}}$ increases. Therefore, an increase in the total energy consumption is expected due to an increase in the energy consumption during the report phase. Since $P_{\mathrm{FA}, \mathrm{FC}}$ is the same for both SNRs, the throughput $D$ is kept unchanged. Therefore, from (18) it can be concluded that the energy efficiency decreases when $\gamma_{\mathrm{FC}}$ increases.

\section{Comparison between the New System and the System of [22]}

This section presents a comparison between our fusion scheme and the efficient decision fusion based on the OR rule proposed in [22]. The reason for this comparison is the similarity between them in one specific configuration of the scheme of [22].

The OR rule is a particular case of $K$-out-of- $M$ rule when $K=1$. In this case, the FC will decide for the presence of the PU if at least one of the SUs decides in favor of $H_{1}$. To reduce the energy consumption, a censoring rule is applied in [22] during the report phase. Therefore, only those SUs that have local decisions in favor of $H_{1}$ will be able to transmit their decisions, using a continuous wave signaling. To increase the bandwidth efficiency, these SUs transmit 
the same signal at the same time slot. If the FC detects the signal in that time slot, this indicates that one or more SUs have local decision $H_{1}$. Therefore, a global decision $H_{1}$ will be the final decision, based on the OR rule. Otherwise, it will be $H_{0}$.

Assuming that $U$ is associated to the duration of the time slot for signaling, the $u$-th discrete-time instant $(u=0,1, \ldots, U-1)$ of the continuous wave used to transmit the local decision from the $i$-th SU to the FC, $i=1,2, \ldots, M$, is formulated as [22]

$$
x_{i}(u)=\left\{\begin{array}{lll}
0 & ; & \left(H_{L, i}=H_{0}\right) \\
A_{i} \exp \left(j \omega_{c} u+\theta_{i}\right) ; & \left(H_{L, i}=H_{1}\right)
\end{array},\right.
$$

where $H_{L, i}$ is the local decision of the $i$-th SU, $A_{i}$ and $\theta_{i}$ are, respectively, the peak amplitude and phase of the continuous wave.

In [22], power and phase controls were proposed to increase the detection performance of the secondary network. Then, to carry out a fair comparison with our proposed system, the power and phase controls shall not be considered. In this case, named $\mathrm{DF}_{n, n}$ in [22], the amplitude and phase components assume the values $A_{i}=1$ and $\theta_{i}=0$.

For a pure additive white Gaussian noise (AWGN) reporting channel, the received signal at the FC at the $u$-th instant is

$$
y(u)=\sum_{i=0}^{M^{*}} x_{i}(u)+w(u),
$$

where $M^{*}$ is the number of SUs that declared the presence of the PU signal.

The test statistic $T$ proposed in [22] is formed according to

$$
T=\frac{1}{U}\left|\sum_{k=0}^{U-1} y(u) \exp \left(-j \omega_{c} u\right)\right|^{2} .
$$

The FC decides for the presence of the PU signal if the test statistic is greater than a pre-defined decision threshold $\lambda_{G}$. Otherwise, the FC decides for the absence of the PU signal. In [22], the threshold $\lambda_{G}$ is chosen to satisfy the target global false alarm probability $P_{\mathrm{FA}, \mathrm{FC}}^{(\mathrm{T})}=0.1$.

Both the new scheme proposed here and the OR rule scheme proposed in [22] use the censoring concept with simultaneous transmission of local decisions. The main difference between these two schemes lies in the decision fusion strategy. In the new scheme it is possible to use the $K$-out-of- $M$ rule, while the scheme in [22] is limited to the OR rule that is based on a sort of energy detector implemented at the FC. In other words, our decision fusion scheme can estimate how many SUs have transmitted their local decisions, which renders our scheme more generic.

In [22], the signal-to-noise ratio (SNR) is defined as $\gamma_{\mathrm{FC}}^{\prime}=\left(K \alpha_{i} / \sigma_{w}^{2}\right)$, where $\alpha_{i}$ is the report channel gain between the $i$-th SU and the FC, and $\sigma_{w}{ }^{2}$ is the variance of the zero-mean white Gaussian noise at the input of the FC. For AWGN report channels $\alpha_{i}=1$.

In our scheme, the SNR is defined as $\gamma_{\mathrm{FC}}=E_{\mathrm{b}} / N_{0}$, where $E_{\mathrm{b}}$ is the average energy per transmitted bit and $N_{0}=2 \sigma_{w}^{2}$ is the unilateral noise power spectral density. 
At the end of the report period, in the new scheme we have a single sample $r$ at the FC, which can be seen as a sample at a correlator output. On the other hand, in [22] there are $U$ samples $\{y(u)\}$ which are used in the computation of the test statistic $T$ given in (21).

In order to perform a fair comparison, we have defined that the duration of the time slot, defined by $U$, must be equal to the time needed for the correlator to output the sample $r$, leading to $E_{\mathrm{b}}=U$ (recall that $A_{i}=1$ ). Therefore, the following mapping must be made:

$$
\frac{E_{b}}{N_{0}}=\frac{U}{2 \sigma_{w}^{2}} \quad \therefore \quad \gamma_{\mathrm{FC}}=\frac{\gamma_{\mathrm{FC}}^{\prime}}{2} .
$$

Numerical results in terms of ROC curves regarding these two schemes are shown in Fig. 7. To plot these results we have arbitrarily assumed $N=100, M=5, K=1$ (OR rule) and $K=\lceil M / 2\rceil=3$ (majority voting rule) for the new scheme, $\gamma_{\mathrm{SU}}=-5 \mathrm{~dB}$ and $\gamma_{\mathrm{FC}}=0 \mathrm{~dB}$ (left) and $8 \mathrm{~dB}$ (right), leading to $\gamma_{\mathrm{FC}}^{\prime}=3 \mathrm{~dB}$ and $11 \mathrm{~dB}$. The results for $K=M=5$ (AND rule) were omitted, since in this case the ROC curves for the AND rule exhibit a bounding (saturation) effect in the probability of detection and the ROC curves for the system proposed in [22] exhibit the bounding effect in the probability of false alarm, making it difficult a fair performance comparison. Each value on the simulated ROC curves was obtained from 500,000 Monte Carlo events. Each event corresponds to computing $P_{\mathrm{D}, \mathrm{SU}}$ and $P_{\mathrm{FA}, \mathrm{SU}}$ for a given decision threshold and signal-to-noise ratio.
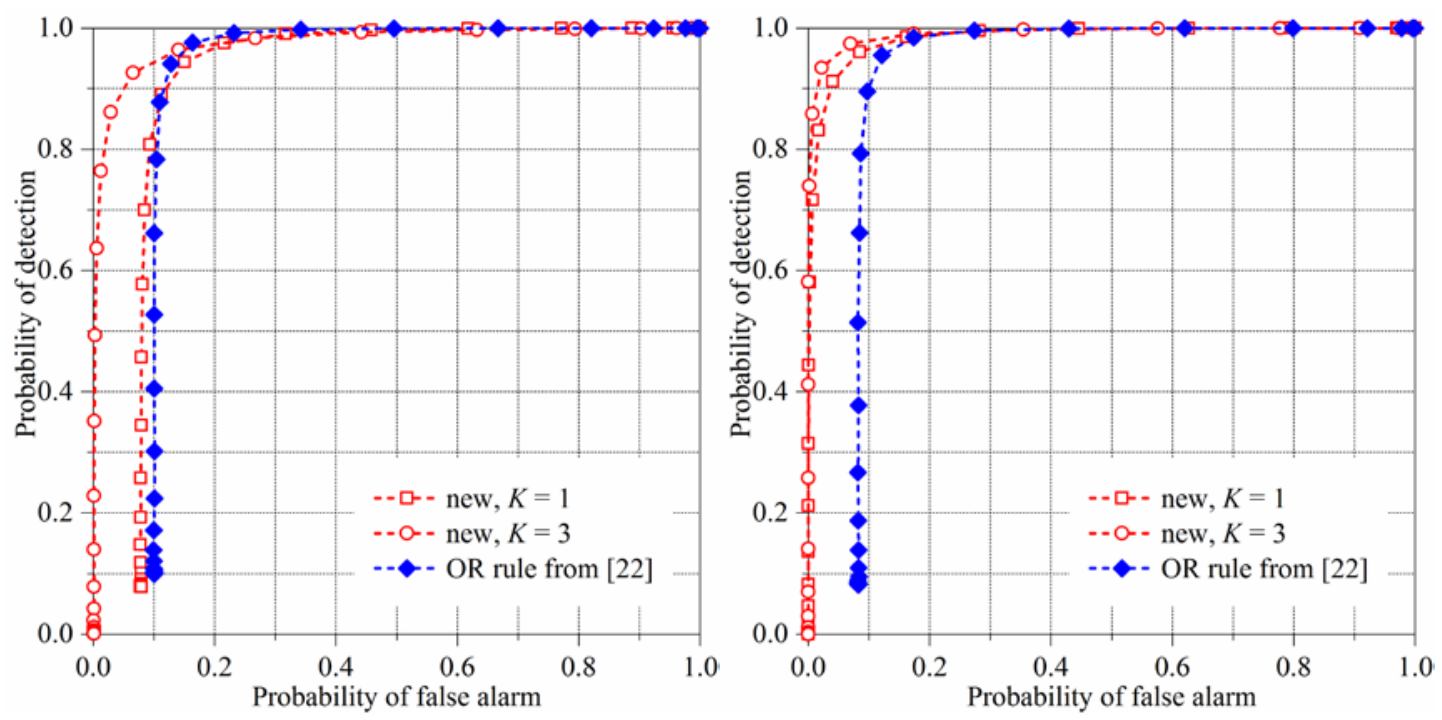

Fig. 7. Global probabilities of detection and false alarm for the new and the OR rule scheme of [22]. $\gamma_{\mathrm{FC}}=0 \mathrm{~dB}$ left, $\gamma_{\mathrm{FC}}=8 \mathrm{~dB}$ right. First rule adopted for determining target metrics.

In the case of the system proposed in [22], the SUs decisions in favor of $H_{1}$ are sent to the FC using continuous wave. In this case, a unitary vector with length $U$ is used to reproduce the signal $x_{i}(u)$ during the report round. At the FC, all $\left\{x_{i}(u)\right\}$ samples are added according to (20), with the noise $w(u)$ generated as $\sim N\left(0, \sigma_{w}{ }^{2}\right)$. The value of the test statistic $T$ is computed using (21), and then compared with the threshold $\lambda_{G}$ previously calculated to yield the target global false alarm $P_{\mathrm{FA}, \mathrm{FC}}^{(\mathrm{T})}=0.1$. The final decisions at the FC are used for computing false alarms (if 
the PU is off) and detection (if the PU is on) rates, which are the estimates of the associated probabilities. Repeating the above procedure, by varying the decision threshold at the SUs, the ROC curves are traced out.

According to the results, it is possible to notice that the performances of the OR rule of [22] and the new schemes are similar when $\gamma_{\mathrm{FC}}=0 \mathrm{~dB}$ and $K=1$. In this situation, the energy consumption of these two schemes is the same during the report and the opportunistic transmission phases. Therefore, it is correct to state that, in this condition, the energy efficiency will be the same in both schemes. When $\gamma_{\mathrm{FC}}=0 \mathrm{~dB}$ and $K=3$, the performance of the new scheme is superior to the OR rule scheme of [22]. In this situation, the energy consumption during the report phase is equal to one another for both schemes. However, the achievable throughput of the new scheme is greater than OR rule scheme of [22]. Therefore, the energy efficiency can be increased in the proposed scheme by only changing the decision fusion rule to majority-voting, contrasting with the limitation of the OR decision fusion rule of [22]. For values of $\gamma_{\mathrm{FC}}>0 \mathrm{~dB}$, it can be noticed that the new scheme outperforms the OR rule of [22] for both $K=1$ and $K=3$. This can be observed in Fig. 7 (right), where the numerical results for simulations with $\gamma_{\mathrm{FC}}=8 \mathrm{~dB}$ are shown. In this situation, for the OR rule of [22], it is observed a significant increase (from approximately 0.7 to 0.9 ) in the global detection probability at the point where the global false alarm is 0.1 . However, the increased performance of the proposed scheme is considerably higher. Therefore, it can be concluded that, for $\gamma_{\mathrm{FC}}=8 \mathrm{~dB}$, the energy efficiency of the new scheme is greater than the one achieved by the OR rule scheme of [22] for $K=1$ and $K=3$, due to the higher achievable throughput in the first scheme.

\section{Conclusions}

The new fusion scheme proposed in this article provides a large increase in the energy efficiency when compared with the scheme proposed in [23], maintaining the bandwidth efficiency. A significant energetic gain was observed when values of practical interest are considered for the global false alarm and detection rates at the fusion center. Besides being more energy-efficient, the new scheme can achieve better performances than the reference one, in terms of ROC, mainly when the signal-to-noise ratio in the report channels is low, a situation that is of great practical appeal. It was also verified that the expressions derived in this article provide results with good agreement with simulations, for any of the parameters analyzed. Moreover, it was demonstrated that the new decision fusion scheme exhibits an energy efficiency that is equal to or greater than the energy efficiency of the decision fusion strategy proposed in [22], a conclusion that can be drawn due to the fact that the performance of the new scheme is better than or approximately equal to the one unveiled by the scheme of [22] in terms of ROC, for the system configurations under which both schemes can be fairly compared. The contributions reported in this article allows for the implementation of a cooperative spectrum sensing system that simultaneously cares about the bandwidth and the energy efficiency, aspects of paramount importance in wireless communication systems. 


\section{References}

[1] J. Mitola III and G. Q. Maguire Jr., "Cognitive radio: making software radios more personal," IEEE Personal Communications, vol. 6, no. 4, pp. 13-18, August, 1999. Article (CrossRef Link).

[2] F. Khan and K. Nakagawa, "Comparative study of spectrum sensing techniques in cognitive radio networks," in Proc. of World Congress on Computer and Information Technology, pp. 1-8, June, 2013. Article (CrossRef Link).

[3] I. F. Akyildiz, B. F. Lo and R. Balakrishnan, "Cooperative spectrum sensing in cognitive radio networks: A survey,” Elsevier Physical Communication, vol. 4, no. 1, pp. 40-62, March, 2011. Article (CrossRef Link).

[4] X. Chen, H.-H. Chen and W. Meng, "Cooperative communications for cognitive radio networks: from theory to applications," IEEE Communications Surveys and Tutorials, vol. 16, no. 3, pp. 1180-1192, March, 2014. Article (CrossRef Link).

[5] X. Hong, J. Wang, C.-X. Wang and J. Shi, "Cognitive radio in 5G: a perspective on energy-spectral efficiency trade-off,” IEEE Communications Magazine, vol. 52, no. 7, pp. 46-53, July, 2014. Article (CrossRef Link).

[6] C. Sun, W. Zhang and K. Letaief, "Cooperative spectrum sensing for cognitive radios under bandwidth constraints," in Proc. of Wireless Communications and Networking Conference, pp. 1-5, March, 2007. Article (CrossRef Link).

[7] Y. Zou, Y.-D. Yao and B. Zheng, "A cooperative sensing based cognitive relay transmission scheme without a dedicated sensing relay channel in cognitive radio networks," IEEE Transactions on Signal Processing, vol. 59, no. 2, pp. 854-858, February, 2011. Article (CrossRef Link).

[8] Y. Zou, Y.-D. Yao and B. Zheng, "A selective-relay based cooperative spectrum sensing scheme without dedicated reporting channels in cognitive radio networks," IEEE Transactions on Wireless Communications, vol. 10, no. 4, pp. 1188-1198, April, 2011. Article (CrossRef Link).

[9] S. Mitsuya, K. Kobayashi, T. Yamazato and M. Katayama, "Improvement of sequential-test-based cooperative spectrum sensing systems in band limited control channels," in Proc. of International Symposium on Communications and Information Technologies, pp. 968-973, October, 2010. Article (CrossRef Link).

[10] Q. Zou, S. Zheng and A. Sayed, "Cooperative sensing via sequential detection," IEEE Transactions on Signal Processing, vol. 58, no. 12, pp. 6266-6283, December, 2010. Article (CrossRef Link).

[11] H. Vu-Van and I. Koo, "A sequential cooperative spectrum sensing scheme based on cognitive user reputation," IEEE Transactions on Consumer Electronics, vol. 58, no. 4, pp. 1147-1152, November, 2012. Article (CrossRef Link).

[12] L. Chuan-qing and W. Zhi-ming, "Adaptive weighted algorithm of cooperative spectrum sensing in cognitive radio networks," in Proc. of International Communication Conference on Wireless Mobile and Computing, pp. 121-126, November, 2011. Article (CrossRef Link).

[13] C. Sun, W. Zhang and K. Ben, "Cluster-based cooperative spectrum sensing in cognitive radio systems," in Proc. of IEEE International Conference on Communications, pp. 2511-2515, June, 2007. Article (CrossRef Link).

[14] A. Kozal, M. Merabti and F. Bouhafs, "Spectrum sensing-energy tradeoff in multi-hop cluster based cooperative cognitive radio networks," in Proc. of IEEE Conference on Computer Communications Workshops, pp. 765-770, April, 2014. Article (CrossRef Link).

[15] B. Wang, Z. Bai, Y. Xu, P. Dong and K. Kwak, "Dynamical clustering cooperative spectrum sensing with bandwidth constraints in CR systems," in Proc. of 16th International Conference on Advanced Communication Technology, pp. 244-248, February, 2014. Article (CrossRef Link).

[16] G. Nie, Y. Wang, G. Li and M. Xu, "Sensing-throughput tradeoff in cluster-based cooperative cognitive radio networks: A novel frame structure," in Proc of 75th IEEE Vehicular Technology Conference, pp. 1-5, May, 2012. Article (CrossRef Link). 
[17] S. Althunibat and F. Granelli, "Energy-efficient reporting scheme for cooperative spectrum sensing," in Proc. of 18th IEEE International Workshop on Computer Aided Modeling and Design of Communication Links and Networks, pp. 28-32, September, 2013. Article (CrossRef Link).

[18] S. Althunibat and F. Granelli, "Novel energy-efficient reporting scheme for spectrum sensing results in cognitive radio," in Proc. of IEEE International Conference on Communications, pp. 2438-2442, June, 2013. Article (CrossRef Link).

[19] S. Maleki and G. Leus, "Censored truncated sequential spectrum sensing for cognitive radio networks,” IEEE Journal on Selected Areas in Communications, vol. 31, no. 3, pp. 364-378, March, 2013. Article (CrossRef Link).

[20] S. Althunibat and F. Granelli, "An objection-based collaborative spectrum sensing for cognitive radio networks,” IEEE Communications Letters, vol. 18, no. 8, pp. 1291-1294, August, 2014. Article (CrossRef Link).

[21] J. So, “Cooperative spectrum sensing for cognitive radio networks with limited reporting,” KSII Transactions on Internet and Information System, vol. 9, no. 8, pp. 2755-2773, August, 2015. Article (CrossRef Link).

[22] K. Umebayashi, J. J. Lehtomaki, T. Yazawa and Y. Suzuki, "Efficient decision fusion for cooperative spectrum sensing based on OR-rule," IEEE Transactions on Wireless Communications, vol. 11, no. 7, pp. 2585-2595, July, 2012. Article (CrossRef Link).

[23] C. H. Lim, "Resource-efficient transmission for report channel in cooperative spectrum sensing," Electronics Letters, vol. 50, no. 16, pp.1171-1173, July, 2014. Article (CrossRef Link).

[24] D. A. Guimarães and G. P. Aquino, "Resource-efficient fusion over fading and non-fading reporting channels for cooperative spectrum sensing,” Sensors, vol. 15, no. 1, pp. 1861-1884, 2015. Article (CrossRef Link).

[25] C. Rago, P. Willett and Y. Bar-Shalom, "Censoring sensors: a low-communication-rate scheme for distributed detection,” IEEE Transactions on Aerospace and Electronic Systems, vol.32, no.2, pp.554-568, April, 1996. Article (CrossRef Link).

[26] D. Cabric, A. Tkachenko, and R. W. Brodersen, "Experimental study of spectrum sensing based on energy detection and network cooperation," in Proc. of the 1st International Workshop on Technology and Policy for Accessing Spectrum, pp.12-es, 2006. Article (CrossRef Link).

[27] S. Atapattu, C. Tellambura and H. Jiang, "Energy detection based cooperative spectrum sensing in cognitive radio networks,” IEEE Transactions on Wireless Communications, vol. 10, no. 4, pp. 1232-1241, April 2011. Article (CrossRef Link). 


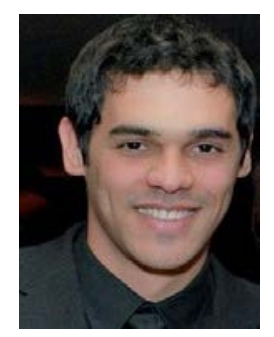

Guilherme Pedro Aquino received the M.Sc. degree in telecommunications engineering from National Institute of Telecommunications (Inatel), Brazil, and is currently a lecturer and researcher at the Inatel. His main research interests lie in the study of cooperative spectrum sensing techniques for cognitive radio applications.

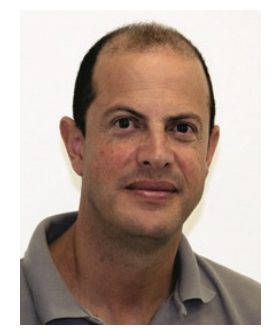

Dayan Adionel Guimarães has a $\mathrm{PhD}$ in Electrical Engineering from State University of Campinas (Unicamp), Brazil, and is currently a lecturer and researcher at the National Institute of Telecommunications (Inatel), Brazil. His research interests lie in the the general aspects of digital transmission and mobile communications. He is currently working on spectrum sensing techniques for cognitive radio applications, multi-slope propagation models and applications of convex optimization in communications.

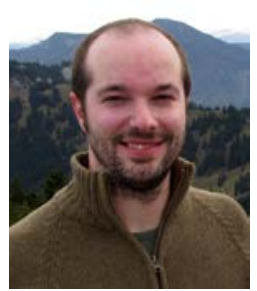

Marco Cattaneo has a PhD in Mathematics from ETH Zurich, Switzerland, and is currently a lecturer in the Department of Mathematics of the University of Hull, UK. His main research interests lie in the study of reasoning and decision making under uncertainty, with particular focus on the likelihood approach to statistics. 